\title{
A New Test Method of Circuit Breaker Spring Telescopic Characteristics Based Image Processing
}

\author{
Huimin Huang, Feifeng Wang, Yufeng Lu, Xiaofei Xia, and Yi Su \\ Electric Power Research Institute of Guangxi Power Grid Co., Ltd, Nanning, Guangxi 530023, China
}

\begin{abstract}
This paper applied computer vision technology to the fatigue condition monitoring of springs, and a new telescopic characteristics test method is proposed for circuit breaker operating mechanism spring based on image processing technology. High-speed camera is utilized to capture spring movement image sequences when high voltage circuit breaker operated. Then the image-matching method is used to obtain the deformation-time curve and speed-time curve, and the spring expansion and deformation parameters are extracted from it, which will lay a foundation for subsequent spring force analysis and matching state evaluation. After performing simulation tests at the experimental site, this image analyzing method could solve the complex problems of traditional mechanical sensor installation and monitoring online, status assessment of the circuit breaker spring.
\end{abstract}

\section{Introduction}

The reliability of high-voltage circuit breaker operation is vital to the protection and control of the power grid. According to the statistics, most of the failures of highvoltage circuit breakers are faults of the operating mechanism, and the spring of the operating mechanism is an important component of the circuit breaker. In normal operation, the spring of high voltage circuit breaker mainly bears changing load or is in long-term tight state, so most of the failure modes are reduction in rigidity or fatigue failure by Dai Wen-fang [1]. Accidents of circuit breaker failure due to sudden breakage caused by long-term fatigue work of the spring often occur. Therefore, it is of great significance to study the fatigue condition monitoring of spring in operating mechanism.

At present, the monitoring of the high voltage circuit breaker operating mechanism focuses more on the overall mechanical performance of the operating mechanism. Through direct or indirect measurement of moving contact opening and closing stroke curve, switching coil current, etc. There are few studies on spring monitoring and status evaluation methods. Some new circuit breakers have pressure sensors installed at the bottom of the spring in the manufacturing process, but most circuit breakers can only rely on manual regular inspection, which is not only time-consuming but also labor - consuming as follows [2] and [3]. The function of the general spring fatigue tester is to calculate the fatigue curve of the spring, which needs to be tested after disassembling the circuit breaker spring, so the on-line analysis of the spring force and deformation state of the circuit breaker operating mechanism cannot be achieved.
Zhao Si-yang [4] proposes that the decrease of the rigidity of the switching energy-storing spring of the circuit breaker will cause the eigenfrequency of the spring to decrease. Using this point, the acceleration sensor is used to detect the state of the spring energy storage, but it does not leave the range of contact measurement, and different circuit breakers need to be designed with different installation equipment, which is not conducive to popularization.

This study uses a high-speed camera to capture the deformation of the operating mechanism spring when the circuit breaker is opened and closed. Based on the deformation characteristics of the spring during the operation of the circuit breaker, the first quarter of the movement is used as the motion recognition target. A normalized cross correlation (NCC) algorithm with adaptive ROI region is proposed by Zhao shu-tao [2], which can continuously adjust the ROI region. This method not only guarantees the matching accuracy, but also can obtain the parameters of the spring expansion characteristics of the circuit breaker more quickly and efficiently.

\section{Circuit Breaker Spring Telescopic Deformation Detection}

In this paper, the high-speed camera is used to replace traditional contact sensors, which obtains the image sequences of the deformation of the circuit breaker operating mechanism springs during the opening and closing process. Image matching technology are used to detect the spring deformation. This "non-contact" circuit breaker spring test method effectively solves the problem of complex installation of the traditional sensor.

\footnotetext{
$\overline{\text { * Corresponding author: 397163797@qqq.com }}$
} 
When the circuit breaker coil current reaches the set threshold, the high-speed camera is triggered to capture the action or energy storage process of the circuit breaker operating mechanism spring; Image preprocessing are used to improve the quality of the image to be analyzed, which reduces the interference of field light change and camera electronic noise on the captured images, and influence on the recognition accuracy of the target on the spring; then a suitable region of interest (ROI) is set, and the NCC algorithm is used to capture the motion of the coil closest to the motion end and the position of the fixed-end coil. The positional relationship between the two above is used to determine the real-time length and deformation of the spring, and various characteristic parameters such as the free length of the spring, the effective number of turns, the deformation, the maximum stroke, and the wire diameter are calculated [5] and [6], which lays the foundation for spring mechanical analysis, fatigue condition monitoring and defect fault diagnosis of the circuit breaker operating mechanism. The specific detection process is shown in Fig. 1.

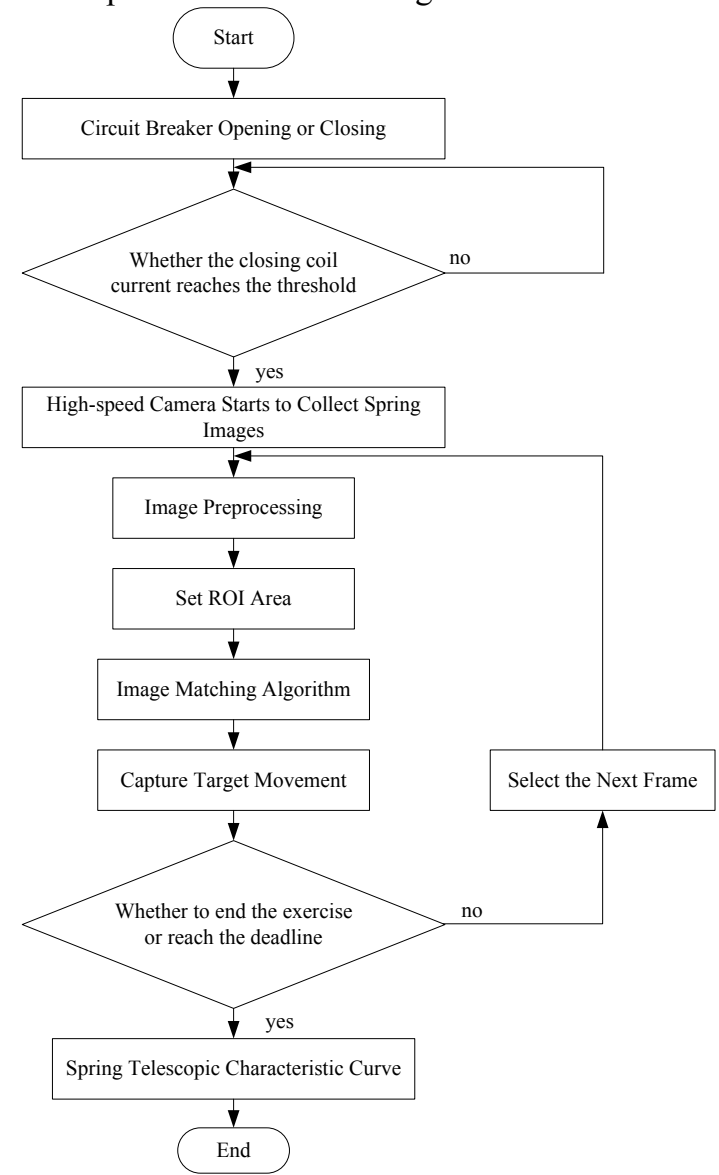

Fig. 1. Flow Chart of Circuit Breaker Spring Telescopic Deformation Detection

\section{Spring Image Matching Algorithm}

\subsection{Normalized Cross-Correlation Algorithm}

Normalized cross-correlation matching algorithm is a classical matching algorithm based on image grayscale, often written as NCC algorithm [8]. This method matches the template image and the original image one by one and calculates the cross-correlation values of the template image and the image to be matched to determine the degree of matching. The position of the search point with the largest cross-correlation value determines the position of the template image in the image to be matched. The algorithm is as follows.

$$
\begin{aligned}
& \operatorname{NCC}(m, n)=\frac{\sum_{x} \sum_{y}(G(x, y)-\bar{G}(x, y))(F(x+m, y+n)-\bar{F}(x+m, y+n))}{\sqrt{\sum_{x} \sum_{y}(G(x, y)-\bar{G}(x, y))^{2} \sum_{x} \sum_{y}(F(x+m, y+n)-\bar{F}(x+m, y+n))^{2}}} \\
& \bar{G}(x, y)=\sum_{x=1}^{r} \sum_{y=1}^{s} G(x, y) /(r \times s) \\
& \bar{F}(x+m, y+n)=\sum_{x=1}^{r} \sum_{y=1}^{s} F(x+m, y+n) /(r \times s)
\end{aligned}
$$

Here we use $\mathrm{F}$ to represent the original image, whose pixel value is $R \times S$; the template image is $\mathrm{G}$, whose pixel value is $r \times s . \mathrm{F}(\mathrm{x}+\mathrm{m}, \mathrm{y}+\mathrm{n})$ is the gray value of $(\mathrm{x}+\mathrm{m}, \mathrm{y}+\mathrm{n})$ point in the original image $\mathrm{F}$, while $\mathrm{G}(\mathrm{x}, \mathrm{y})$ is the gray vaule of $(x, y)$ point of the template image $G$. $(m, n)$ is the position of the top left vertex of the template image $G$ in the original image $F$ when searching. When the coordinates of the top left corner vertex of the template image $G$ is $(m, n)$, the point $G(x, y)$ in the template image $\mathrm{G}$ is corresponding to the point $\mathrm{F}(\mathrm{x}+\mathrm{m}, \mathrm{y}+\mathrm{n})$ in the original image $\mathrm{F}$. Through the above formula (1), the value of NCC between the template image $G$ and the sub-image of the original image $F$ under its coverage are calculated. When the correlation values of all positions throughout the original image $\mathrm{F}$ and the template $\mathrm{G}$ is obtained, the sub-image which is correspond to the maximum value is the destination image, and its position is the coordinate to be determined.

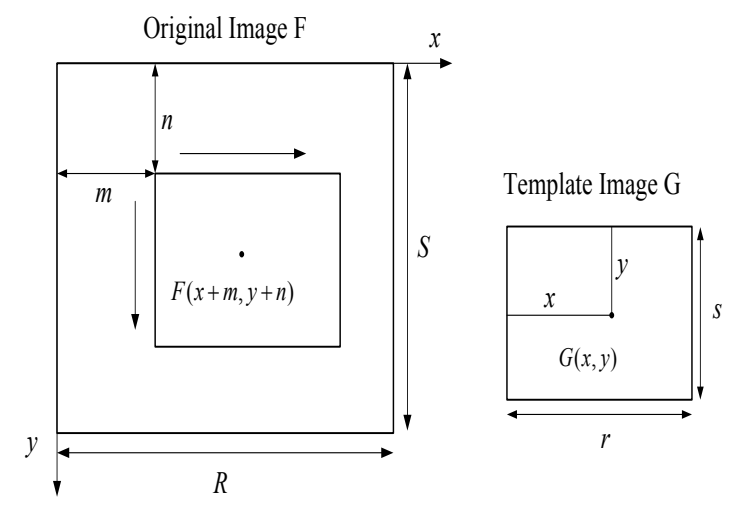

Fig. 2. Principle of NCC algorithm

Although the NCC algorithm has high matching accuracy, the disadvantages such as large amount of calculation and slow operation speed affect the application in moving target recognition of the circuit breaker. In order to solve these problems, the following improvement is adopted to reduce the amount of calculation and increase the efficiency to meet the realtime requirements of on-line monitoring.

\subsection{Adaptive ROI Area}


Before the image matching starts, a suitable area is set, ie, the target motion range, is selected as the region of interest (ROI), which is generally a rectangle along the pixel direction. In the process of image processing, only the ROI area needs to be analyzed. The approach can not only greatly decrease the computational complexity of the image analysis process, but also eliminate the interference of the structure outside the region. In order to further reduce that area of the ROI region and reduce the amount of calculation, the position of the ROI region is adjusted from the target position of the previous frame when the image to be matched of the next frame is processed, starting with the process of the second frame image. The specific adjustment method is as follows.

The center of the new ROI area is decided by target position of the previous frame. The length and width of the new frame satisfy the following relationship.

$$
L_{i}=k l+c+\delta_{i-1}
$$

The value of $\mathrm{k}$ is determined according to the spring deformation direction. It is 1 when to non-main direction of deformation, and become 1.2 when to the main direction of deformation (telescopic direction). 1 is the length and width of the template image. $\mathrm{c}$ is a fixed value of 10 to 20 pixels, (here take 10 pixels). $\delta_{i}$ is the distance moved from the previous frame compared to the target. This method is equivalent to prediction, which greatly limits the size of ROI region and improves the matching efficiency

\section{Circuit Breaker Spring Flexibility Measurement Example}

A test platform is set up at the experimental site. The closing process of a ZN65-12 high-voltage circuit breaker is selected as the subjection. The coil current was set to $0.5 \mathrm{~A}$ in order to trigger the high-speed camera to capture the motion of the circuit breaker operating mechanism spring at a velocity of 3,500 fps. The initial frame of the target motion is searched and then the fixedend pixel coordinates is recorded as the initial point A, which is used to conveniently calculate the actual working length and movement speed of the spring in the circuit breaker deformation process. The coil closest to the moving end of the spring, $\mathrm{B}$, is selected as the moving target which needs to be identified in the image. Finally, the target is identified by the adaptive ROI region image matching algorithm to obtain the pixel position in each frame of the image, which represent the actual working position of the operating mechanism spring.

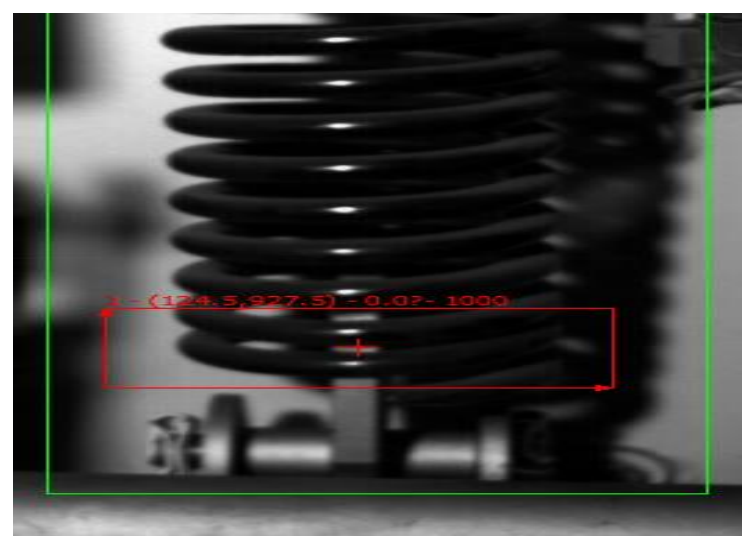

a) Spring Fixed End

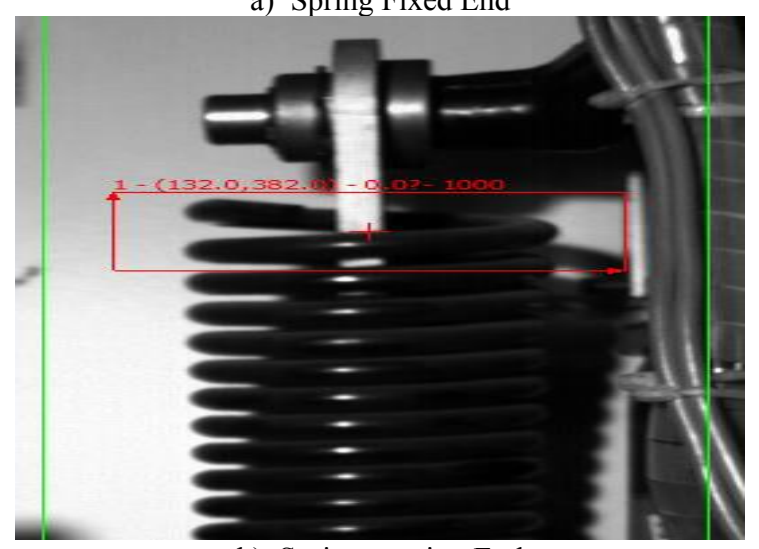

b) Spring moving End

Fig. 3. Circuit Breaker Spring Image Matching Result

The distance between the target position B and the initial point $A$ in each frame is calculated to plot the displacement-time curve and velocity-time curve of the moving target during the deformation of operating mechanism spring. The speed of the operating mechanism spring is the point-by-point slope value of the displacement-time curve. The result is as follows.

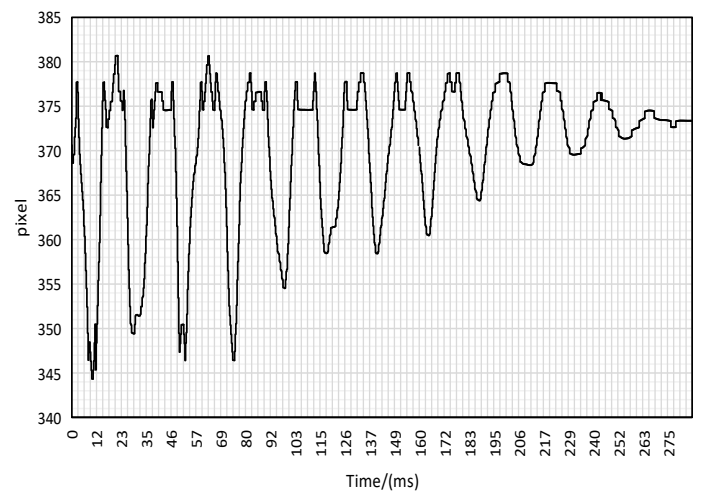

Fig. 4. Target Displacement-Time Curve (Stretch Direction) 


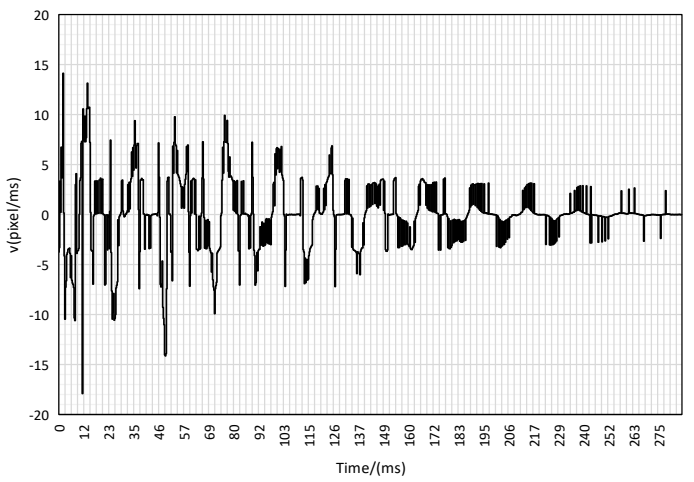

Fig. 5. Target Speed-Time Curve (Stretch Direction)

It can be seen from the figure that the spring is continuously reciprocating during the circuit breaker closing processed by Li Pengfei [7]. According to the motion curve of the circuit breaker spring, the initial frame target pixel position $s_{0}$ was determined to be 370.62, and the fixed-end pixel position $s$ was 927.5. The initial spring height $H_{t 0}$ was calculated to be 556.9. The final working height $H_{t}$ was calculated as 554.1 which is determined by the difference between the ending frame target pixel postion $s_{t}$ at 373.36 and the fixed-end pixel position $s$ (the numerical units above are all pixels).

The maximum $\mathrm{x}$-coordinate value of the intersection point between the straight line $\left(y=s_{t} \pm \varepsilon\right.$ ) and the spring displacement time curve is taken as the spring stretching time t. $\varepsilon$ is used to judge the maximum amplitude of the spring still in expansion or compression motion, (default can take 0 2 pixels, one pixel value is taken in this experiment). Such setup is to eliminating the action caused by the identification error due to the too small spring stretching range. Finally, the spring stretching time $\mathrm{t}$ was calculated to $274 \mathrm{~ms}$.

The difference between the upper and lower bounds of the spring displacement-time curve is calculated as the maximum movement amplitude which was obtained to be 36.4 pixels. Through the displacement-time curve, the times that the coordinates of the first coil of the spring crossed its final coordinate during spring retraction is counted as the number of radial reciprocations. It was obtained to be 26 times. Finally, the maximum operating speed of the circuit breaker spring was 16 pixels/ms by analyzing the target speed-time curve.

Table 1. Circuit Breaker Operation Mechanism Spring Extension Characteristics.

\begin{tabular}{|c|c|c|}
\hline Parameter Name & Unit & Value \\
\hline Initial Working height & Pixels & 556.9 \\
\hline Final Working height & Pixels & 554.1 \\
\hline Telescopic time & ms & 274 \\
\hline Maximum stroke & Pixels & 36.4 \\
\hline
\end{tabular}

\begin{tabular}{|c|c|c|}
\hline No. of reciprocations & times & 26 \\
\hline Maximum speed & Pixels/ms & 16 \\
\hline Telescopic Frequency & $\mathrm{Hz}$ & 47.4 \\
\hline
\end{tabular}

Processing 1000 frames of image by utilizing the adaptive ROI area's NCC algorithm takes about $22.8 \mathrm{~s}$. Compared to using the NCC algorithm alone, the same process required $69.4 \mathrm{~s}$ to complete. Obviously, the former algorithm is much faster on the speed of calculation. On the premise of not to decline the calculation accuracy, it greatly accelerates the calculation speed and saves the analysis time, and provides a new method for the online detection of the spring telescopic characteristics on the circuit breaker.

\section{Conclusion}

1) Image processing technology is utilized to analyze the spring telescopic characteristics of the circuit breaker operating mechanism. It can reflect spring expansion and contraction status in the energy storage and release process and extract the spring deformation parameters. This work is a new method of circuit breaker spring detection.

2) The NCC algorithm has a high matching accuracy and is suitable for use in the spring detection of the circuit breaker operating mechanism. However, a large amount of calculation requires stronger hardware support. The improved method improves the speed by 3 times without reducing the calculation accuracy, which is conducive to the promotion of the algorithm.

3) Identifying the circuit breaker spring fatigue based on computer vision technology is a kind of "non-contact" test method. It can effectively solve problems in sensor installation, such as time-consuming and laborconsuming. It has a broad application prospect in the circuit breaker spring online monitoring and electrification test.

\section{References}

1. W.F.Dai, S.Y.Zhao, G.Zeng, etal. Prediction of state of closing spring energy storage based on characteristic parameters of circuit breakers[J]. Instrument Technology and Sensors,08 (2015) .

2. S.T.Zhao, C.J.Wu,M. Li, etal. Study on testing method of circuit breaker mechanical characteristics based on NCC-P-S optimization algorithm $[\mathrm{J}]$. Proceedings of the CSEE,37 (2017) .

3. G. Guo,P.F. Li, J.Li, etal. Status Monitoring of Energy Storage Springs for Circuit Breaker Closing $[\mathrm{J}]$. Instrument Technology and Sensors, 04 (2015).

4. S.Y.Zhao, A.B.Wang, etal. Evaluation of Spring Energy Storage State of Circuit Breaker Operating Mechanism Based on Fuzzy Synthetic Evaluation[J].High Voltage Apparatus, 52 (2016). 
5. J.H.Sohn, S.K.Lee, S.O Kim, etal.Comparison of Spring Models for Dynamic Analysis of a High Voltage Circuit Breaker with a Spring Operating Mechanism $[\mathrm{J}]$. Mechanics Based Design of Structures and Machines,36(2008).

6. W.S.Yoo,S.O.Kim,J.H.Sohn. Dynamic analysis and design of a high voltage circuit breaker with spring operating mechanism $[\mathrm{J}]$. Journal of Mechanical Science and Technology, 21(2007).

7. P.F.Li,W.J.Zhou,G.Zeng,etal.Dynamic characteristics of the closing spring of high voltage circuit breaker and detection method of energy storage state $[\mathrm{J}]$.Transactions of China Electrotechnical Society, 31(2016).

8. A.Pourmohammad, S.Poursajadi, Karimifar S. Scene matching NCC value improvement based on contrast matching[C]/Proceedings of the 2013 8th Iranian Conference on Machine Vision and Image Processing.Zanjan:IEEE,(2013).

9. Y. Fouda, K.Ragab. An efficient implementation of normalized cross-correlation image matching based on pyramid $[\mathrm{C}] / /$ Proceedings of 2013 International Joint Conference on Awareness Science and Technology and Ubi-Media Computing. Aizuwakamatsu:IEEE,(2013).

10. L.J.Sun, X.G.Hu,Y.Ji.Mechanical fault classification of high voltage circuit breakers based on support vector machine $[\mathrm{J}]$. Transactions of China Electrometrical Society, 08(2006).

11. J.A.Martinez-Velasco,J.Magnusson.Parametric analysis of the hybrid HVDC circuit breaker[J]. International Journal of Electrical Power \& Energy Systems, 84(2016). 